\title{
MÁS ALLÁ DE LA ENFERMEDAD: LAS PRERROGATIVAS DE LA BIOMEDICINA ACTUAL
}

Prof. Dra. María Lucrecia Rovaletti

Institución: "Departamento de Humanidades Médicas" de la Facultad de Medicina de la Universidad de Buenos Aires e "Instituto de Bioética y Humanidades Médicas" de la Fundación Mainetti - Consejo Nacional de Investigaciones Científicas y Técnicas. (Argentina).

Correspondencia: Juramento $14534^{\circ}$ piso Dpto. 29

(1428) Capital Federal Argentina

Fono/Fax: (+ 54 11) 4 783-9110

E-mail: mlrova@psi.uba.ar 



\title{
MÁS ALLÁ DE LA ENFERMEDAD: LAS PRERROGATIVAS DE LA BIOMEDICINA ACTUAL
}

\author{
Prof. Dra. María Lucrecia Rovaletti ${ }^{(*)}$
}

\section{Resumen}

Ante los problemas planteados por las nuevas tecnologías, la ética médica tradicional no está capacitada para responder satisfactoriamente. Actualmente, las investigaciones y aplicaciones experimentales biotecnológicas son cada vez más numerosas y artificiosas, siguiendo un deseo y una constancia que no conoce límites y cuyo desequilibrio o meta-estabilidad constituye su único e infatigable motor. La búsqueda de conocimiento se borra frente a la implacable "lógica de la eficacia". Este problema se agrava puesto que la actual revolución biológica (Bernard), a diferencia de la revolución terapéutica que afectaba al ejercicio de la medicina en relación al individuo, alcanza a la humanidad en tanto que especie. Pero, ¿controlar las actividades científicas no sería equivalente a restringir la adquisición de los conocimientos fundamentales?

A dominios nuevos corresponden responsabilidades nuevas. Frente al viejo humanismo finalista y paternalista, un nuevo humanismo apela a la responsabilidad. Por esto el "principio de responsabilidad" (Jonas) no adquiere un sentido ético sino en la medida que cada individuo se descubre como autor, de un modo u otro, de los cambios de gran amplitud de fuerzas que él no controla. En una época donde la ciencia se ha hecho cada vez más operatoria, activa y manipuladora, es decir donde el saber es también poder y actuar, la reflexión filosófica tiene que dirigirse a una nueva Razón Práctica.

Palabras clave: Biotecnología, Biomedicina, Control social de la ciencia, Investigación con propósitos cognitivos

\section{Resumo}

Ante os problemas apresentados pelas novas tecnologias, a ética médica tradicional encontra-se incapacitada para respondê-los satisfatoriamente. Atualmente, as investigações e aplicações experimentais biotecnológicas são cada vez mais numerosas e sofisticadas, porém cativas de desejo e constância que desconhecem limites e cujo desequilíbrio constitui seu único e infatigável motor. A busca do conhecimento turvase frente à implacável "lógica da eficácia". Este problema apresenta-se grave já que a revolução biológica (Bernard) diferentemente da revolução terapêutica que afetava o exercício da medicina no prisma individual, alcança a humanidade enquanto espécie. Questiona-se, então, controlar as atividades científicas não significa restringir a aquisição de novos conhecimentos fundamentais?

Os novos domínios da ciência exigem novas responsabilidades. Frente ao velho humanismo finalista e paternalista, o novo humanismo apela para a responsabilidade. Assim, o "princípio da responsabilidade" (Jonas) somente adquire um sentido ético na medida em que cada indivíduo descobre-se, de alguma maneira, como autor das mudanças de grande magnitude de forças que ele próprio não controla. Num tempo em que a ciência torna-se cada vez mais operativa e manipuladora, ou seja, quando o saber também significa poder, a reflexão filosófica tem que dirigir-se para uma nova Razão Prática.

Palavras chaves: Biotecnologia, Biomedicina, Contrôle social da ciência, Investigação com propósitos cognitivos.

\footnotetext{
Investigador Independiente del CONICET. Docente Adscripta a cargo del Curso Superior de Antropología Médica y Bioética de la Facultad de Medicina y Profesora Regular de Psicología Fenomenológica de la Facultad de Psicología, Universidad de Buenos Aires.

Este trabajo ha sido realizado en el marco del Proyecto "Ética y vida urbana contemporánea. Transformaciones, desplazamientos y nuevos sentidos de los conceptos éticos”, PIP-CONICET No 4560/96, 1997-2000. E-mail: mlrova@psi.uba.ar
} 


\section{Abstract}

In the presence of the problems posed by new technologies, traditional medical ethics is not able to respond satisfactorily. At the present time, research and biotechnological experimental applications are more and more numerous and artificial, following a desire and a constancy that ignores limits and whose unbalanced condition or meta-stability constitutes its unique and tireless motive. The search for knowledge vanishes in front of the implacable "logic of efficacy". This problem becomes worse because present biological revolution (Bernard), in contrast to therapeutical revolution -which affected the medical practice related to the individual- affects humanity as a species. But, wouldn't the control of scientific activities be equivalent to restrict the acquisition of fundamental knowledge?

To new domains correspond new responsibilities. Opposite to the old humanism founded on finality and paternalism, a new humanism appeals to responsibility. Thus, the "principle of responsibility" (Hans Jonas) has an ethical sense only when each individual discovers himself as author, in one sense or the other, of long-range changes and of forces he does not control. In a time in which science has become more operative, active and manipulative -that is to say where knowledge is also power and actingphilosophical thinking has to be directed to a new Practical Reason.

Key words: Biotechnology, Biomedicine, Social control of science, Research of cognitive purpose

\section{Résumé}

Devant les problèmes posés par les nouvelles technologies l'éthique traditionnelle n'est pas qualifié pour répondre d'une manière satisfaisante. Actuellement les recherches et les applications expérimentales biotechnologiques sont de plus en plus nombreuses et artificieuses, suivant un désir et une persévérance qui ne connait pas de limites et dont l'équilibre constitue son seul et infatigable moteur. La recherche de la connaissance disparait face à l'implacable "logique de l'efficacité". Ce problème s'aggrave car l'actuelle révolution biologique (Bernard), au contraire de la revolution therapeutique qui transformait la medicine au regard de l'individu atteint l'humanité en tant qu'espèce.

Mais, le fait de contrôler les activités scientifiques ne serait-il pas équivalent à restreindre l'adquisition des connaissances fondamentales?

Des nouvelles responsabilité correspondent à de nouveaux domaines. Vieux humanisme finaliste et paternaliste, un nouveau humanisme fait appel à la responsabilité.

C'est pour sa que le "principe de responsabilité" (Jonas) n'acquiert un sens éthique que dans la mesure que chaque individu se découvre à lui même tel qu'auteur, d'une facon ou d'une autre, des changements importants de forces qu'il ne contrôle pas. Dans une époque dans laquelle la science est devenue de plus en plus opératoire, active et manipulatrice, c'est à dire où sont lieés le savoir, le pouvoir et l'agir, la réflexion philosophique doit être dirigée vers une nouvelle Raison Pratique.

Mots clés: Biotéchnologie, Biomédecine, Contrôle social de la science, Recherche à de fins cognitives. 
"Las nuevas tecnologías desarrolladas en el ámbito de la Biología y la Medicina han llevado a que la sociedad se cuestione con insistencia sobre sus repercusiones, tanto para el modelo de vida actual como para el futuro de la humanidad. Ante el rápido ritmo de los descubrimientos biotecnológicos se suscita una actitud ambivalente que va desde la ciega confianza en el progreso técnico hasta el temor a la posibilidad de que los científicos ‘jueguen a dioses' y pongan en el envite la supervivencia del planeta” (1, p.11).

\section{La biotecnología: una herencia de la modernidad}

La constante transformación de la realidad y las innumerables intervenciones sobre la vida del hombre traducen de modo preciso el ideal de liberación de la humanidad propio del Iluminismo. El programa de la Modernidad, de expandir siempre más allá los límites -ya para modificar la realidad objetiva ya para perfeccionar la capacidad de acción humana- se acrecienta ahora en la tecnociencia actual. Esta, en su acelerado avance, ya no se contenta con alcanzar un punto de equilibrio o de saturación respecto a los objetivos prefijados, sino que produce otros nuevos que acaban convirtiéndose en nuevas y posteriores motivaciones (2).

Como la ciencia para lograr sus propios objetivos teóricos necesita de una tecnología cada vez más refinada, desaparece ahora la "vieja y noble" distinción entre "teoría" y "práctica". Se produce entonces una profunda socialización del "síndrome tecnológico" (3) al ser puesto al servicio de las necesidades comunes. Ni la ciencia ni la tecnología pueden ser juzgadas como entidades independientes, y si ellas aparecen a menudo como potencias benéficas para unos y maléficas para otros, ello se debe a procesos socio-históricos mucho más amplios.

Después de la época moderna en más, no tiene sentido ni teórica ni históricamente continuar pensando según esta prospectiva dual -homo sapiens/homo faber- ...el momento técnico y aquél especulativo están íntimamente conexos, contribuyendo a la construcción práctica y teórica, de la imagen de la realidad (4, p.49-50).

Por otra parte, como cada innovación científico-tecnológica se difunde rápidamente tanto en el plano del conocimiento como en el de la apropiación práctica, la tecnociencia sufre la presión de la competencia no sólo por los beneficios que ofrece sino también por el poder, la seguridad y el prestigio que otorga.

De este modo, todo descubrimiento y toda realización tecnológica nueva se presenta aureolada de la sacrosanta palabra de "progreso", y sostenida por una visión cuasi-utópica de una "vida cada vez mejor" a la cual la técnica pareciera haber respondido $(5,6)$.

Dado que hoy en día la técnica alcanza a casi todo lo que concierne a los hombres vida y muerte, pensamiento y sentimiento, acción y padecimiento, entorno y cosas, deseos y destino, presente y futuro-, se ha convertido en un problema tanto central como apremiante de toda la existencia humana sobre la tierra... (3, p.15).

En el ámbito de la biomedicina, las investigaciones y aplicaciones experimentales biotecnológicas (7) son cada vez más numerosas y artificiosas, siguiendo un deseo y una constancia que no conoce fronteras y cuyo desequilibrio o metaestabilidad constituye su único e infatigable motor. Las demandas aumentan con la misma rapidez que los medios inventados para satisfacerlas. La comunicación en materia de salud-enfermedad, sostenida 
desde la asimetría del "saber médico" y constantemente distorsionada por la "racionalidad" de la ganancia, se dirige a un terreno necesitado de ayuda, pero sobre todo, altamente predispuesto.

En otros tiempos, todo progreso médico se emprendía desde una perspectiva terapéutica, paliativa, o aún cognitiva, pero siempre preparatoria de prácticas posteriores, a fin de que aparezcan aceptables y convenientes, respetando los límites y equilibrios naturales. Los discursos y las prácticas de la medicina competían juntas para el mejoramiento de la salud, y la eliminación de la enfermedad.

Aquel utópico título con que Vesalio nombrara en 1543 a su libro De humani fabrica corporis pareciera ahora haberse hecho realidad: es el "fetichismo de la tecnología" como dice Portillo (8). Sin embargo, en la medida que la tecno-medicina actual reduce al hombre a una máquina sofisticada (9) de piezas sustituibles, desprovista de valor o digna de interés solamente por su utilidad práctica, dejando de lado la relación íntima consigo mismo o con el prójimo (10, p.275), se corre el peligro de convertirlo en un animal desnaturalizado y también en una mercancía con la que se puede lograr una buena ganancia. La cultura occidental mientras endiosa al cuerpo sataniza la enfermedad y la muerte: el cuerpo es el chivo expiatorio médico-mitológico moderno. "La enfermedad, la vejez y la muerte son disfunciones y desperfectos que el médico, técnico de los cuerpos-máquina, podrá remediar" ${ }^{\text {’i }}(8)$.

Como señalara Heidegger ya en 1947, en Brief über den Humanismus (11) el error del biologismo es no haber comprendido que "el cuerpo del hombre es algo esencialmente distinto que el de un organismo animal". Nos preguntamos entonces ¿cómo podrá la biomedicina actual en su voluntad

i Thomas, J.V.: La muerte, una lectura cultural, Barcelona, Paidós, 1991. Citado por J. Portillo, o.c. demiúrgica satisfacer a la ética, si por su misma naturaleza busca fomentar su subversión?

\section{¿Búsqueda de conocimientos o lógica de la eficiencia?}

Bien sabemos que "la realización histórica de un paradigma epistemológico de carácter general" no se lleva a cabo "al margen de la realidad social y cultural en la que se instaura" (12, p.13). La racionalidad tecnológica, como toda forma de racionalidad, debe responder a un conjunto de exigencias determinadas por la situación social, política, económica, de su tiempo.

La "tecnociencia" como expresión acentuada de la voluntad de dominio del hombre moderno, exige la disponibilidad y la utilidad pragmática de los conocimientos; más aún, no puede ya ser comprendida sólo en términos de una "lógica de los conocimientos", pues está invadida por la competencia desenfrenada, la rentabilidad industrial y la especulación bursátil. Como dice George Ferné, "la ciencia se ha convertido en una mercadería" (13, p.435).

En este sentido, la biomedicina y las biotecnologías no pueden escapar de esta situación en la medida que sufren el ataque frontal del capital que busca movilizar a través de ellas montos millonarios. El mundo de los descubrimientos biológicos y de la innovación biomédica se ha convertido en un campo donde se habla cada vez en términos de mercado, de portafolios de patentes, estrategias de grupos y alianzas a escala transnacional. Hasta los eventos científicos parecieran constituir una buena ocasión para orientar y hasta reorientar el tema de investigación de los científicos. Las fronteras del conocimiento se borran frente a la implacable "lógica de la eficacia", donde la salud cuenta menos que la "voluntad de dominio técnico": la investigación está más orientada a la producción que al conocimiento. 
En nuestra sociedad, lo "normal" lo constituye la salud, y la enfermedad está asociada al fracaso, al rol productivo y autónomo. Esto permite que la publicidad y promoción de nuevos productos biotecnológicos que combaten la "ruptura del orden normal", aprovechando su dimensión simbólica, logren un impacto muy superior del que se podría esperar si la respuesta fuera absolutamente racional y lógica.

Este doble discurso de la eficacia técnica y la eficacia simbólica (8), se difunde a través de los medios masivos de comunicación, para "vender" a la opinión pública, incluso a los médicos, no sólo productos farmacéuticos que prometen efectos terapéuticos cada vez más maravillosos, sino también una aparatología sofisticada cuyas imágenes o gráficas resultan a menudo incomprensibles incluso para muchos médicos. Finalmente uno puede ofertar hasta "noticias provenientes de célebres científicos" en cualquier tipo de programas periodísticos o de telenovelas.

La opinión pública, incluidos los médicos, devienen espectadores deslumbrados que reciben más o menos pasivamente el bombardeo de una información indiscriminada. El nuevo escenario de la publicidad médica comienza a ser dominada por la tecnología ultrasofisticada, al mismo tiempo que la farmacología acaba descentrando la antigua relación personal entre el médico y el paciente. Si la tendencia al consumismo propia de la post-modernidad ha creado objetos que quitan hasta el insomnio, la angustia, las úlcera y un largo etcétera, "qué mejor y más 'normal' que esperar todo de los expertos y consumir mercancías preciosas" (14, p.108). Y si el médico no satisface la demanda, ésta se desplaza sobre el medicamento que deviene el "objeto bueno", mientras la frustración y falta de confianza convierten al médico en "objeto malo".

\section{Metas y tensiones en la medicina actual}

Pocas transformaciones de la vida humana han sido tan grandes como aquellas producidas por las ciencias biomédicas y por la práctica misma de la medicina ${ }^{\text {ii. }}$. A pesar de ello, razones científicas, económicas, sociales y políticas colocan a la medicina actual en constante tensión ya sea por los éxitos alcanzados como por los fracasos admitidos (15). La investigación biomédica no se presenta ni más ni menos indemne a las lógicas del mercado que de cualquier otra actividad. La promoción de la salud y de la enfermedad no son un discurso homogéneo. ¿Será tal vez que la medicina se ha alejado de su propia identidad?

- Los adelantos de la medicina y el perfeccionamiento de sus servicios han servido de poderoso estímulo para la lucha a favor de la salud, pero han creado expectativas que producen a veces demandas públicas irreales.

En tanto que el deseo por el progreso conduce a mayor conocimiento e innovación, también incrementa -paradójicamente-el nivel de descontento con cada situación presente, que tiende a ser vista como inadecuada a la luz de las futuras posibilidades (16, p.10-11).

- La biomedicina ha multiplicado el tipo de intervenciones para aliviar el sufrimiento y salvar a los enfermos, pero son aún soluciones incompletas. Gracias a la biotecnología, se puede mantener funcionando enfermos

\footnotetext{
ii Los avances en el campo de la salud no se deben sólo a los conocimientos biológicos y a las técnicas curativas sino a la creación y generalización de los seguros de salud, que permitieron el acceso a la salud de todos. Por ello, no se puede acreditar a la ciencia y a la técnica beneficios que no son solamente los suyos. Desconocer que la ciencia y la técnica son obra de un mundo social implica mistificar las explicaciones científico-tecnológicas.
} 
Más allá de la enfermedad: las prerrogativas de la biomedicina actual - M. Rovaletti

graves y con características irreversibles, pero también se puede generar con ello dilemas éticos en torno a la suspensión del tratamiento, o puede ser cuestionada por la futilidad de sus prácticas.

- Si bien la alta tecnología diagnóstica y terapéutica ha logrado éxitos en muchos casos cercano a lo milagroso, ella ha elevado enormemente el costo de la medicina y de la asistencia sanitaria. El productivismo encubre una serie de artificios, los cuales pone a nuestra disposición y satisfacción sin que se verifique si éstos nos permiten vivir mejor, y si no conllevan daños a cada uno y a la humanidad en su totalidad.

- A pesar que los desarrollos biotecnológicos han ampliado las opciones y posibilidades humanas, la medicina actual corre el peligro de trans-formarse en una mera colección de actos y técnicas que pueden ser utilizadas sin otra restricción que la económica. Se abandonan los sistemas defensivos propios y se los transfiere a los aparatos y a los fármacos. Aparece aquello que Baudrillard (17) llamará "patologías de tercer tipo", es decir nuevas enfermedades de cuerpos superprotegidos por el escudo artificial, médico o informático. "El hombre se vuelve vulnerable a la medicina" (8).

Habría que preguntarse si con ello hemos transformado las simples necesidades en demandas fantasmáticas a las cuales ninguna satisfacción les puede otorgar una respuesta plena. Como cuestiona Testart (18), ¿se trata sólo de un mero desplazamiento del deseo, o hay además una dialéctica entre la ganancia y la ciencia, propia de la era tecnológica?

\section{La tecno-ciencia incriminada, entre una "heurística del temor" y la "ideología del progreso indefinido"}

Cuando se hace objeto de incriminación a la "tecno-ciencia", la acusación se dirige menos a su producción que a las leyes del mercado que distorsionan a menudo sus objetivos, la sustraen a nuestro dominio, y hasta la vuelven contra nosotros mismos. "Sucede entonces que lo más humano se conmuta en lo más inhumano" (19, p.277).

Heidegger en Die Frage nach der Technik (20) muestra que el hombre al estar sometido "al cálculo y a la organización", acaba reduciéndose a la "materia prima" más importante; y por eso se piensa que un día, sobre las bases de investigaciones químicas, se edificarán fabricas para la producción artificial de la misma. Esta transformación de la biología en biofísica, supone que el hombre puede ser producido, de acuerdo a parámetros determinados, como cualquier objeto técnico. Pero la autoproducción del hombre conlleva el peligro de su autodestrucción. Precisamente, la biofísica no puede saltar fuera de ella misma, ni ese salto tampoco puede hacerlo la política porque ella misma vive hoy en la dimensión de la ciencia, por ella y para ella (21). La reflexión heideggeriana nos muestra ya, que el peligro no nos viene de la técnica sino por la técnica.

Pero este cuestionamiento no implica un resentimiento a la ciencia pues constituiría una nueva manifestación de la intolerancia al progreso que otrora marcara a la historia de la ciencia. Tampoco busca restringir la adquisición de los conocimientos fundamentales, ni renovar con vehemencia los ataques ético-filosóficos contra una biociencia que quiere "jugar el rol de Dios", ni implantar una heurística del temor (22) pues este alarmismo diaboliza en vez de analizar, excomulga en lugar de solidarizar (19).

Como no se puede hablar del poder de la ciencias (23) sin dejar de subrayar también la responsabilidad y el poder de los científicos 
que han contribuido ampliamente a la creciente dependencia de los individuos respecto a las técnicas, nos preguntamos si se busca controlar la "ciencia" o "controlar sus actividades científicas". ¿No lleva esto a concebir el control como una polémica vigilancia externa, a alimentar sospechas recíprocas que generan una interminable querella al progreso que impide una fructífera cooperación entre las partes?

Se trata de hacer entrar la biomedicina dentro del marco de los derechos humanos y no adaptar los derechos humanos a la biomedicina. Sólo se podrá avanzar hacia una real solución del problema si se trabaja en el acercamiento de la cultura científica y los valores humanos, planteando nuevas alianzas entre las tareas científicas y las demandas humanas: no al mero freno ético que se oponga al desarrollo científico, y sí a un esfuerzo sostenido que integre la dimensión ética a la dinámica científica. La técnica y la ciencia en general tienen que asumir la responsabilidad profunda de las lógicas deshumanizantes que se asocian a la producción impetuosa de saberes: la búsqueda de conocimientos muy a menudo se borra frente a la necesidad de dominio a la cual éstos le sirven de coartada.

Frente al des-fondamiento de las referencias tradicionales, frente a una era llamada "individualista" y a la carencia vertiginosa de apropiación social nos preguntamos: ¿en nombre de qué criterios es posible fijar unos marcos y límites a las investigaciones y desarrollos tecnológicos? ¿cómo lograr un consenso que pueda responder a estas urgencias?

\section{Una razón práctica para la responsa- bilidad cósmica}

A diferencia de la revolución terapéutica que afectaba al ejercicio de la medicina respecto al sujeto individual, la actual revolución biológica alcanza a la humanidad en tanto que especie (24). El hombre es capaz de acciones cuyos efectos nocivos son de dimensión cósmica, y es por ello que la sociedad está confrontada a elecciones de las cuales depende la humanidad de mañana.

En las ciencias de la vida, el dominio de la reproducción (en las tres áreas de la contracepción, inseminación artificial y fecundación in vitro) o el dominio de la herencia a través de la genética o el dominio del sistema nervioso constituyen un nuevo modo de actuar que puede modificar la evolución de las especies, más aún de modificar la especie humana.

A dominios nuevos corresponden responsabilidades nuevas. Frente al viejo humanismo finalista y paternalista, un nuevo humanismo apela a la responsabilidad.

Frente a un concepto de "responsabilidad" en cuanto imputabilidad por las acciones del pasado, Jonas postula el "principio de responsabilidad" $(3,25)$, de una responsabilidad dirigida a un futuro que supera aquél de las consecuencias previsibles. Es una responsabilidad sin responsabilidad asignable, ante una humanidad futura esencialmente frágil. El hombre actual ha devenido peligroso para el hombre, poniendo en riesgo la vida y el habitat donde vive.

El "principio de responsabilidad" no adquiere un sentido ético sino en la medida que cada individuo se descubre como autor de un modo u otro de los cambios de gran amplitud de fuerzas que él no controla. Como las prácticas individuales devienen actualmente prácticas institucionales, este problema no sólo concierne hoy a los individuos que son responsables de los actos científicos-tecnológicos, sino a toda la sociedad.

"La especie humana es el único producto de la evolución que sabe que ha evolucionando y que quiere continuar evolucionando" (26, p.17) apostando a una evolución cultural en la cual nuestras acciones puedan justificarse éticamente (27). El hombre ha tenido que recorrer sinuosos e 
imprescindibles caminos para convertirse en la especie homo sapiens, y está constantemente urgido a encontrar nuevos equilibrios que movilizan a su vez nuevos riesgos e incertezas. "La dimensión tecnológica de la existencia humana es ya una dimensión ontológica"(12, p.203). ¿Cómo entonces la sociedad puede renunciar a tales avances, ante las sorpresivas dificultades que se puedan plantear?

Si la sobrevivencia de la especie humana constituye un objetivo ampliamente aceptado, más aún si "el derecho a la humanidad a existir" (3) deviene un nuevo imperativo, "el problema de la ciencia" se transforma en el problema ético al que hay que descubrir el apropiado curso de sus acciones reorientando nuestro modo de pensar y de comportarnos, y revisando antiguas y arraigadas creencias. Si la ciencia como proyecto occidental ha devenido no sólo conocer sino también poder y actuar, si "la razón técnica significa además la culminación de la dinámica histórica abierta por la Modernidad siglos atrás, y quizás también una de su principales herencias para el futuro próximo"(12, p.211), entonces la reflexión filosófica tiene que dirigirse a una nueva Razón Práctica. La novedad del imperativo moral del "principio de responsabilidad cósmica" de Hans Jonas (3), excede el respeto de la persona humana de Kant en tanto ética centrada en la projimidad y la reciprocidad. Precisamente porque:

La responsabilidad en la era tecnológica, se extiende tan lejos como lo hacen nuestros poderes en el espacio y en el tiempo, y en las profundidades de la vida (28, p.261).

\section{Referencias}

1. Casado M, (ed ). Bioética, derecho y sociedad. Madrid: Trotta; 1998.

2. Ladrière J. Les enyeux de la rationalité: le défi de la science et de la technologie aux culture. Paris: Aubier-UNESCO;1977.

3. Jonas H. Técnica, medicina y ética: la práctica del principio de responsabilidad. Barcelona: Paidós; 1997.

4. Pessina A. Bioetica: l'uomo sperimentale. Milano: Bruno Mondadoni; 1999.

5. Del Tacca M, (comp). L'etica nella ricerca biomedica. Roma: La Nuova Italia Scientifica;1997.

6. De Siqueira JE. Desafíos éticos de la medicina de alta tecnología. Cuadernos del Programa Regional de Bioética 1997; 4:103-21.

7. Escudé J. Una ética para la era tecnológica. Cuadernos del Programa Regional de Bioética 1997;5: 63-83.

8. Portillo J. El discurso médico. Relaciones 1995; 137:11-3.

9. Rovaletti M L. El saber biomédico y la metáfora mecanicista. Perspectivas Bioéticas en las Américas 1998; 6: 24-39.

10. Le Breton D. Le chair à vif; usages medicaux et mondaines du corps humaines. Paris: Métailie; 1993.

11. Heidegger M. Brief über den humanismu. Berna: Francke; 1947.

12. Queraltó R. Racionalidad tecnológica y mundo futuro: la herencia de la Razón Moderna. Seminarios de Filosofía 1998; 11:203-19.

13. Ferné G. La science, une nouvelle marchandise. La Recherche 1989; 208: 428- 35.

14. Páez D. El sentido social de la enfermedad. Revista Occidente 1985; 47:103-14.

15. Bianca M. Scienza, etica e bioetica. Firenze: Angelo Ponte Corboli Editore; 1999.

16. The Hastings Center. The goals of medicine: setting new priorities. Hastings Center Report 1996; 26 ( Special Suppl 6):1-48.

17. Baudrillard J. La transparencia del mal. Ensayo sobre los fenómenos extremos. Barcelona: Anagrama; 1991.

18. Testart J. Entretien sur l' Bioéhtique. In: F Lenoir. Le temps de la responsabilité; entretiens sur l'éthique. Paris: Fayard; 
1991. p. 69-75.

19. Séve L. Pour une critique de la raison bioéthique. Paris: Odile Jacob; 1994.

20. Heidegger M. Die Frage nach der Technik. In: Vorträge und Aufsätze. Neske: Pfullingen; 1954. p.21.

21. Heidegger M. Questions IV. Paris: Gallimard; 1976.

22. Lecourt D. Contre le peur. Paris: Hachette; 1990.

23. Hottois G. Evaluer la technique. Paris: Vrin; 1988.

24. Bernard J. Entretien sur l'Bioéthique. In: F Lenoir. Le temps de la responsabilité; entretiens sur l'éthique. Paris: Fayard; 1991. p.51-4.

25. Rovaletti ML. On cosmic Responsibility. Concordia, International Zeitschrift für Philosophie 1998;33:77-86.

26. Spinsanti S. Bioética global o la sabiduría para sobrevivir. Cuadernos del Programa Regional de Bioética 1998;7:7-17.

27. Sachetti A. L'uomo antibiologico; riconciliare, società e natura. Milano: Feltrinelli; 1999.

28. Ricoeur P. Post-face. In: F Lenoir. Le temps de la responsabilité; entretiens sur l'éthique. Paris: Fayard;1999.p.247-70. 